\section{ENERGY}

\section{EFFICIENT LONGER} LIFE LIGHT-SOURCE

Daray Ltd has released a new premium quality LED light for the dental industry, the QiLite. The QiLite follows on from the resounding success of Daray's BiLite, a unique twin-beamed halogen dental light which was launched over 30 years ago and fast became the dental industry's light of choice. Similar to its predecessor, the QiLite produces a rectangular light-patch, using four LEDs rather than two halogen bulbs to offer advanced Quad Beam technology.

Daray's latest offering uses NviroLED technology and can produce an intense $15 \mathrm{~W}$ beam - equivalent to a $75 \mathrm{~W}$ halogen beam - with a maximum light intensity of 32,000 lux. The QiLite also offers improved energy efficiency and a longer life lightsource, making it an economical purchase given its lifespan.

The QiLite can also accommodate a variety of dental techniques thanks to a much improved triple-axis range of movement and new ergonomic support arms which can be easily manipulated with just one finger. The QiLite can be both ceiling and wall mounted, and is also available as a mobile unit.

The QiLite also incorporates a contact-free inductive switch control which enables the light to operate without being physically touched, further reducing the risk of cross-infection. By simply moving your hand under the head it will switch the light on or off, and moving your hand closer or further away will vary the light intensity.

Daray's QiLite complies with BS EN 60601-2-4-1 and is manufactured at the company's factory in Swadlincote, Derbyshire.

Call 08008048384 or visit www.daray.co.uk.

\title{
RESTORE EXTENSIVE CAVITIES AT CHAIRSIDE
}

GC everX Posterior is a fibrereinforced composite designed to be used as dentine replacement, in conjunction with a conventional composite such as $\mathrm{G}$-ænial Posterior used as enamel replacement. The short fibres of GC everX Posterior will make it a perfect substructure to reinforce any composite restoration in large size cavities. Fibres will also prevent and stop crack propaga- tion through the filling, which is considered to be the main cause of composite failures. Thanks to its unique properties, GC everX Posterior opens new possibilities for restorations of extensive cavities at chairside and is the answer to the growing demand for an economic restorative alternative for big size cavities.

For further information contact GC UK on 01908218999.

\section{ADDRESSING PRACTICE IDIOSYNCRASIES}

Take control of the running of your dental practice with CS Back Office software from Carestream Dental. With this suite of user-friendly tools, all of the key aspects of the business are brought to your fingertips:

- Reporting: monitor performance by running automatic reports on areas ranging from individual members of staff to the number of recalls, new patients or DNAs per month

- Accounting: manage the practice bank account, Purchase Ledger and Nominal Ledger

- Inventory: coordinate petty cash and stock control
- Employment records: organise payroll and store full personnel records, working hours, holidays and sickness absence.

Developed to address the idiosyncrasies of dental practices, CS Back Office software not only helps to improve efficiency but also assists compliance with employee-related CQC outcomes.

Enjoy confidence in the knowledge that you are making sound business decisions thanks to CS Back Office.

For more information, contact Carestream Dental on 0800169 9692 or visit www.carestreamdental.co.uk.

\section{INTUITIVE DIGITAL OPTICAL IMPRESSIONS}

Sirona are excited to announce the launch of the brand new CEREC Omnicam with unrivalled handling, powder-free scanning and precise 3D impressions - all in natural colour. Taking digital optical impressions is now even easier, intuitive and designed for maximum comfort.

CEREC Omnicam's revolutionary design features a completely new, ergonomic handpiece and special optics to guarantee unsurpassed intraoral access. New Colour Streaming allows continuous capture of the oral cavity as well as displaying the 3D data in full colour - an industry first. Operator convenience has been greatly improved by making the system completely powder-free while retaining Sirona's high standards for precision.

The slim, rounded camera tube allows easy rotation of the camera in the intraoral space. The tiny camera tip guarantees the best lens positioning anywhere in the mouth and as the camera moves over teeth, a photorealistic image is displayed in full-colour with crisp clarity and impeccable detail delivering a new level of clinical confidence. There's no need to apply reflective medium making capturing images easier and faster than ever.

CEREC Omnicam perfectly balances all requirements for a chairside CAD/CAM camera with operator convenience, in 3D full colour.

For further information call 08450715040 or email info@ sironadental.co.uk. 